\title{
No Changes in Bone Mineral Density Following Total Knee Arthroplasty Using an All Polyethylene Tibial Component
}

\author{
Alistair M. Ewen, Jill Rae, Artaban J. Jeldi and Frederic Picard \\ Golden Jubilee National Hospital, Clydebank, U.K. \\ Alistair.ewen@gjnh.scot.nhs.uk
}

\begin{abstract}
Polyethylene particles produced in metal backed tibias (MBT) are understood to contribute to bone loss and component loosening. This, and better surgical techniques (including computer navigation) and increasing costs have renewed interest in all poly tibias (APT). We investigated peri-APT bone mineral density (BMD) in patients; expecting to find no differences between two post-operative values.

Patients over 65 years, with $\mathrm{BMI} \leq 37.5$ and no previous joint replacements were recruited to have TKA using the Columbus APT with computer navigation (OrthoPilot). The study cohort $(\mathrm{n}=26)$ had a mean age of 71.9 (sd 4.35), a BMI of 31.2 (sd 3.8). The BMD examinations were performed six weeks and 18 months post-operatively. Six regions-of-interest (ROI) were identified on anterior/posterior and lateral scans. For each ROI at each time point, relative BMD differences (RDs) were determined between limbs and RDs at the two time points were compared.

No differences were found between the two RDs for any ROI. No revisions or complications were reported. At 18 months post-operatively, $78.3 \%$ of the cohort were "very satisfied" with the outcome of their surgery and Oxford Knee Scores improved significantly compared to pre-operatively $(\mathrm{p}<0.005)$. Mean knee range of motion was $102^{\circ}$ (sd $10.7^{\circ}$ ) and mean leg alignment was $2.0^{\circ}$ valgus ( $1^{\circ}$ varus- $6^{\circ}$ valgus $)$.

Results from BMD suggest that implants were well fixated. Patients reported excellent satisfaction and function. We believe that using APTs and computer navigation is a viable and cheaper option to MBT for patients who are less active, have lower BMI and good bone quality.
\end{abstract}

\section{Aims}

It is generally accepted that the tibial component in total knee arthroplasty (TKA) is a prime factor in component failure (Cheng et al., 2012). The use of all polyethylene tibial (APT) components in total 
knee arthroplasty (TKA) procedures all but ceased as a result of bone collapse due to poor implantation technique (Gioe et al., 2010). Mechanical research around this time suggested that a metal backing to the polyethylene bearing would better distribute the load passed through the joint to the cancellous bone (Rand, 1993). There is, however, a belief that the small polyethylene particles produced through backside wear in standard metal backed tibial (MBT) components contributes to the bone loss and subsequent component loosening of these implants (Cheng et al., 2012). It is also thought that the stress shielding caused by the stiff metal backing plate prevents bone regeneration (Gioe, 2010). These, and more accurate surgical techniques (including computer navigation), improvements in material science and increasing costs related to rising number of TKA procedures being performed has renewed interest in APTs. We investigated bone mineral density (BMD) in the proximal tibia of a cohort of patients recruited to have TKA using an APT. We hypothesised that there would be no significant differences in the BMD between two post-operative measures taken six weeks and 18 months post-operatively.

\section{Patients and Methods}

Thirty-two patients were recruited from the clinics of two consultant orthopaedic surgeons. Patients were excluded if they were under the age of 65 , had a BMI of over 37.5 or had a previous hip or knee replacement. Patients with diagnosed osteoporosis were excluded from the study or withdrawn from the study if osteoporosis was diagnosed during the course of their involvement. Recruited patients underwent TKA using the Columbus (APT) (Aesculap AG, Tuttlingen, Germany) with computer navigation using the OrthoPilot (Aesculap AG, Tuttlingen, Germany) system. At six weeks and 18 months post-operatively they had a BMD examination using dual energy x-ray absorptiometry (DXA). Scans were taken of the neck of the femur on the non-operated side to determine overall bone health and of the operated and non-operated knees. Anterior-posterior (AP) and lateral scans of the knees were taken with these repeated to give a total of nine scans per patient per session. Three regions of interest (ROI) were identified on each of the AP and lateral scans during the first of the two scan (Fig. 1), with these replicated on the subsequent scans. BMD values were noted for each region on all of the scans. A mean value of the two repeated measures was used in subsequent analyses. For each region at each time point, a relative BMD difference between the operated and non-operated knee was determined as previously described (Abu-Rajab et al., 2006). Comparisons were made between the first and second BMD measures.

Four patients withdrew from the study and one was found to have osteoporosis following the first bone densiometery examination and was withdrawn from the study. One patient has still to complete the study. This gave a study cohort of 26 patients. The mean age of the cohort was 71.9 (sd 4.35) with a BMI of 31.2 (sd 3.8). There was an equal number of males and females $(n=13)$, while 12 had surgery on the right knee. 


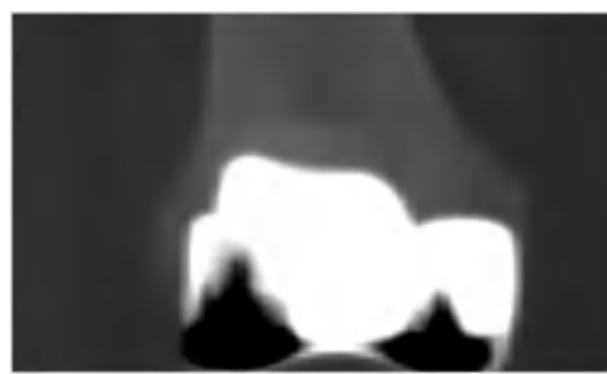

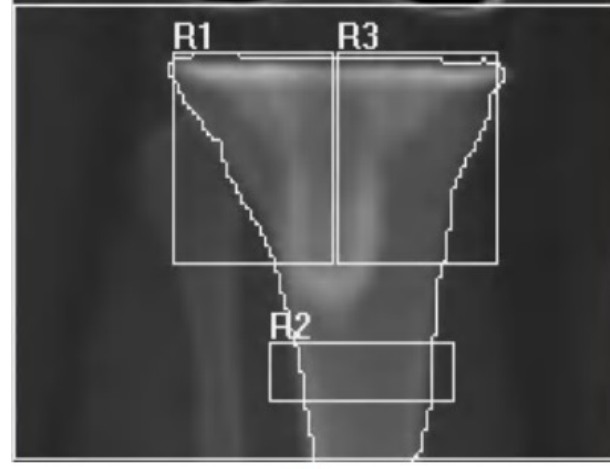

(a)

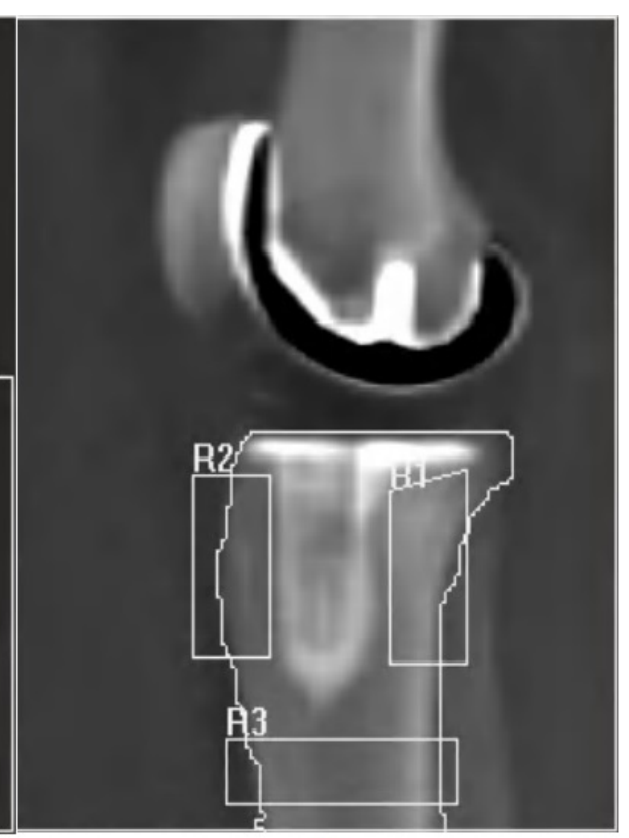

(b)

Figure 1: Anterior-posterior (a) and lateral (b) views from the DXA scans showing the regions of interest; AP R1 lateral proximal tibia, AP R2 proximal tibia distal to the cement mantle, AP R3 medial proximal tibia, Lat R1 posterior tibia, Lat R2 frontal tibia and Lat R3 proximal tibia distal to the cement mantle.

\section{Results}

Sixteen patients had normal bone health as recorded during the first of the DXA examinations with the remaining 10 having osteopenia. A change in bone health was noted between the first and second examinations for three patients. Two of these changed from normal to osteopenic and the other changed from osteopenic to normal. The latter is likely due to differences in the areas sampled. No significant differences were noted for any of the ROIs between the two post-operative DXA examinations using two-tailed, paired samples t-tests (Table 1). 


\begin{tabular}{lccc}
\hline & \multicolumn{2}{c}{ Relative BMD Difference (\%) } & Post-op 2 \\
ROI & Post-op 1 & $77.6(63.42)$ & 0.341 \\
AP R1 & $67.6(47.58)$ & $68.0(75.71)$ & 0.737 \\
AP R2 & $63.9(56.60)$ & $7.2(21.44)$ & 0.402 \\
AP R3 & $3.3(14.79)$ & $-6.5(38.39)$ & 0.105 \\
Lat R1 & $-16.6(24.79)$ & $-29.9(68.76)$ & 0.308 \\
Lat R2 & $-39.7(41.06)$ & $-19.0(19.89)$ & 0.952 \\
Lat R3 & $-18.7(25.35)$ &
\end{tabular}

Table 1: Relative bone mineral density differences between the operated and non-operated knee for regions of interest AP R1 to AP R3 on the anterior-posterior (AP) view and Lat R1 to Lat R3 on the lateral view with comparisons between six week (Post-op 1) and 18 month (Postop 2) DXA scans.

The 18 months post-operative Oxford Knee Score (OKS) was 24 (sd 9.0) and the OKS preoperatively was 40 (sd 7.0)(12-60, best to worst respectively), which is a significant improvement $(p<0.005)$. At 18 months post-operatively, 78.3\% $(n=18)$ of the cohort were very satisfied with the outcome of their surgery on a five point Likert scale, $17.4 \%(n=4)$ were unsure, while one patient $(4.3 \%)$ was dissatisfied. Satisfaction data were not available for three patients. No intra-operative complications were noted and the mean operation time was 78.1 minutes (sd 10.2). Patients in the cohort achieved a mean knee range of motion of $102^{\circ}$ (sd 10.7०). Mean leg alignment at six weeks post-operatively was $2.0^{\circ}$ valgus $\left(1^{\circ}\right.$ varus to $6^{\circ}$ valgus $)$.

\section{Conclusion}

This appears to be the first study to investigate BMD following TKA using an APT. The Columbus APT produced excellent patient satisfaction and functional outcomes. Our patients achieved a slightly better ROM compared to that reported in another study (Campbell et al., 2019). The use of computer navigation ensured that the components were well positioned to ensure good alignment, which may have contributed to the excellent results reported. Up to 18 months postoperatively, no components were revised and there were no other reported complications. A previous study reported a high early failure rate for APT components, but conceded that high BMI was a contributing factor (Alhifzi et al., 2018). Their reported mean BMI for the revised cases (37.6) was higher than our BMI exclusion limit for this study. The results of the DXA assessments show that after 18 months, no significant change in BMD has occurred, suggesting that the implants are well fixated. We believe that TKA using APTs is a viable and cheaper option to the MBT alternative for selected patients who are less active, have lower BMI and good bone quality.

\section{References}

Abu-Rajab, R.B., Watson, W.S., Walker, B., Roberts, J., Gallacher, S.J. and Meek, R.M. (2006). Peri-prosthetic bone mineral density after total knee arthroplasty. Cemented versus cementless fixation. The Journal of bone and joint surgery.British volume, 88(5), pp. 606-613.

Alhifzi, Z.S., Khoja, Y.T., Wood, G.C. (2018). All-polyethylene tibial components in total knee replacement: Early failures. The Journal of Knee Surgery. Oct 3. doi: 10.1055/s-0038-1672124. [Epub ahead of print]. 
Campbell, D., Callary, S., Field, J. and Nilsson, K.G. (2019). All-polyethylene tibial components in young patients have stable fixation; a comparison RSA study, The Knee,

https://doi.org/10.1016/j.knee.2018.12.003. [Epub ahead of print].

Cheng, T., Pan, X., Liu, T. And Zhang, X. (2012). Tibial component designs in primary total knee arthroplasty: should we reconsider all-polyethylene component? Knee surgery, sports traumatology, arthroscopy : official journal of the ESSKA, 20(8), pp. 1438-1449.

Gioe, T.J. And Maheshwari, A.V. (2010). The all-polyethylene tibial component in primary total knee arthroplasty. The Journal of bone and joint surgery.American volume, 92(2), pp. 478-487.

Rand, J.A. (1993). Comparison of metal-backed and all-polyethylene tibial components in cruciate condylar total knee arthroplasty. The Journal of arthroplasty, 8(3), pp. 307-313. 